\title{
La organización de las poblaciones clave ligadas a la transmisión del VIH: una intervención para abatir el estigma; México, 2005-2009
}

\author{
The organization of key populations connected to HIV \\ transmission: an intervention to abate stigma; Mexico, \\ 2005-2009
}

Cuadra-Hernández, Silvia Magali ; Zarco-Mera, Ángel2; Infante-Xibillé, César³; Caballero-García, Marta4.

${ }^{1}$ Socióloga. Doctora en Sociología. Investigadora en Ciencias Médicas, Instituto Nacional de Salud Pública, México.

magali.cuadra@insp.mx

${ }^{2}$ Antropólogo. Maestro en Antropología Social. Investigador honorario, Instituto Nacional de Salud Pública, México.

angel.zarco@insp.mx

${ }^{3}$ Médico cirujano. Doctor en Educación. Investigador en Ciencias Médicas, Instituto Nacional de Salud Pública, México.

cesar.infante@insp.mx

${ }^{4}$ Socióloga. Doctora en Sociología. Investigadora, Universidad Autónoma del Estado de Morelos, campus Oriente, México.

martacg@uaem.mx
RESUMEN Una aproximación cuali-cuantitativa es la base para el análisis de los resultados del proyecto "Vida Digna", cuyo objetivo fue abatir el estigma y la discriminación en el campo de la transmisión del VIH a partir de las acciones realizadas por organizaciones de la sociedad civil durante el período 2005 al 2009, en la región mexicana llamada El Bajío. Los resultados se analizaron en los años 2009 y 2010. Las organizaciones participantes estuvieron compuestas por y para las denominadas poblaciones clave, definidas como grupos vulnerables a infectarse pero también capaces de resistir y controlar la transmisión del VIH, el estigma y la discriminación, que se constituyen en barreras importantes para la búsqueda de atención y en el control efectivo del VIH. Se describen y analizan las acciones y el fortalecimiento de las organizaciones participantes. Resaltan la visibilización de nuevos actores sociales, como las mujeres transgénero y los usuarios de drogas inyectables, y las acciones informativas dirigidas a periodistas, policías y militares para evitar la criminalización y persecución de estos grupos.

PALABRAS CLAVE Organizaciones No Gubernamentales; VIH; Estereotipo; Prejuicio; México.

ABSTRACT A qualitative and quantitative approach forms the base of this analysis of the results of "Vida Digna," a project aimed at abating stigma and discrimination in the HIV transmission field with actions taken by civil society organizations from 2005 to 2009 in the Mexican region of El Bajío. The results were analyzed in 2009 and 2010. The organizations involved were made up of key populations, defined as groups vulnerable to infection but also capable of resisting and controlling the transmission of HIV and the stigma and discrimination that are important barriers in the seeking of care and the achievement of effective HIV control. We describe and analyze the actions taken and the strengthening of the participating organizations. The visibility of new social actors such as transgender women and injecting drug users, as well as informative activities directed at journalists, the police and the military to prevent the criminalization and persecution of these groups, are highlighted.

KEY WORDS Non-Governmental Organizations; HIV; Stereotyping; Prejudice; Mexico. 


\section{INTRODUCCIÓN}

En este artículo se presentan los resultados de las acciones llevadas a cabo para disminuir el estigma y la discriminación hacia grupos sociales relacionados con la transmisión del VIH/sida por organizaciones de la sociedad civil (OSC), a partir de su participación en el proyecto "Vida Digna", durante los años de 2005 al 2009, en la región mexicana conocida como El Bajío, que abarca los estados de Guanajuato, Aguascalientes, San Luis Potosí y Querétaro. Esta zona se ha caracterizado históricamente por su tradición conservadora, sobre todo con relación a las prácticas sexuales de grupos sociales sancionados por las normas morales hegemónicas.

El Censo Nacional de Organizaciones de la Sociedad Civil, elaborado por el Centro Nacional para la Prevención y el Control del $\mathrm{VIH} /$ sida, organismo gubernamental mexicano especializado en la atención de esta enfermedad, señala que existen 318 OSC relacionadas con este tema (1). De ellas, 7 se localizan en Aguascalientes, 3 en Guanajuato, 4 en Querétaro y 2 en San Luis Potosí. Por su parte, el registro de la organización no gubernamental "Amigos contra el Sida" contiene datos de 264 organizaciones, de las cuales 3 se localizan en Aguascalientes, 7 en Guanajuato, 4 en Querétaro y 2 en San Luis Potosí (2).

En el caso del proyecto "Vida Digna", las OSC participantes fueron 18, de las cuales 9 Ilegaron hasta el año 2009. Estas organizaciones estuvieron conformadas por las denominadas poblaciones clave:

El término "poblaciones clave" es usado por la Alianza Internacional contra el $\mathrm{VIH} /$ sida para referirse a aquellos grupos que son clave para la dinámica de la epidemia de VIH. Son grupos de personas afectados por el VIH debido a las normas sociales, a situaciones contextuales y de comportamientos sexuales, o son más propensos a estar en situaciones donde pueden adquirir o transmitir el VIH a causa de sus prácticas. (3 p.3)

Desde el inicio, el proyecto "Vida Digna" propuso la realización de diagnósticos comunitarios para conocer y atender las necesidades específicas de sus poblaciones clave. En todas las organizaciones participantes el diagnóstico señaló que era necesario enfocar acciones de apoyo a personas en circuitos de trabajo sexual, hombres gay y otros hombres que tienen sexo con otros hombres, personas transgénero y usuarios de drogas inyectables (3). Estos grupos, que tienen mayores probabilidades de exponerse directamente al VIH y a la vez de exponer a otros al mismo virus, juegan un papel fundamental para cambiar las dinámicas de la epidemia. El enfoque del proyecto "Vida Digna" consistió, precisamente, en convertir a estas poblaciones en grupos fundamentales para cambiar paradigmas de prevención, acción y barreras en el acceso a los servicios y fortalecer las OSC que coadyuven a estos cambios.

En México, el proyecto fue liderado por el programa Positive Action y la organización Colectivo Sol, la cual forma parte de la Alianza Internacional contra el $\mathrm{VIH} /$ sida, con sede en Inglaterra, que agrupa a OSC que trabajan en torno al control del VIH en todo el mundo.

El estigma y la discriminación forman parte de los ejes culturales que atribuyen cualidades negativas a las personas identificadas con la transmisión del VIH. La percepción negativa de las personas más afectadas por el VIH ha estado ligada a grupos históricamente señalados con atributos descalificadores, como sucede con algunas etnias indígenas, las diferencias de género y/o los grupos que se encuentran en una situación socioeconómica depauperada (4). Los procesos estigmatizadores se constituyen en determinantes de la salud colectiva, dado que son parte del habitus, es decir el espacio de las posiciones sociales que generan y reproducen las prácticas:

\footnotetext{
...distintas y distintivas [...] pero también son esquemas clasificatorios, principios de clasificación, principios de visión y de división, aficiones, diferentes. Establecen diferencias entre lo que es bueno y lo que es malo, entre lo que está bien y lo que está mal, entre lo que es distinguido y lo que es vulgar, etc., pero no son las mismas diferencias para unos y para otros. (5 p.19-20)
}

Esas prácticas determinan la falta de acceso de las poblaciones clave a los servicios de salud, a la posibilidad de prevención y de mitigación del 
daño. Infante et al. (6) documentaron cómo en los servicios estatales de salud mexicanos, los conocimientos, las percepciones y las prácticas de su personal se encuentran impregnados de ideas alejadas de los descubrimientos científicos acerca de la transmisión del VIH. Esta situación, al unirse con las percepciones negativas hacia grupos estigmatizados, provoca violaciones a sus Derechos Humanos como sucede con la confidencialidad del estado de salud y la autonomía para realizarse la prueba; aunque contravenga el discurso oficial y la normatividad que los garantiza. Todo ello provoca maltrato hacia los usuarios infectados con el virus, aplazamiento o cancelación de las citas médicas o de las intervenciones quirúrgicas (6).

La estigmatización es un proceso social que se da en la interacción (7); es un mecanismo de diferenciación y, por lo tanto, es relacional. Respecto a este sistema de relaciones que sirven para establecer diferencias, Bourdieu señala:

Esta idea de diferencia, de desviación, fundamenta la noción misma de espacio, conjunto de posiciones distintas y coexistentes, externas unas a otras, definidas en relación unas de otras, por su exterioridad mutua y por relaciones de proximidad, de vecindad, de alejamiento y asimismo por relaciones de orden, como por encima, por debajo y entre... (5 p.16)

Ello significa que las señales estigmatizadoras siempre se establecen con relación al otro, hacen referencia a "un atributo profundamente desacreditador, [...] lo que en realidad se necesita es un lenguaje de relaciones, no de atributos" (7 p.13). Será entonces una relación entre grupos diferentes y la diferencia se basará en los imaginarios de normalidad y desviación que imperen en ese momento y en ese lugar. Lo que caracteriza al estigma, además de su atribución negativa, es su vocación ejemplarizante a través de la sanción social y el consenso que logra construir a su alrededor.

El estigma sustrae la esencia de lo humano para quien lo porta: “Creemos, por definición, desde luego, que la persona que tiene un estigma no es totalmente humana" (7 p.15). Esto provoca, a su vez, una justificación de las acciones para evitar, invisibilizar o aislar al sujeto social identificado con la anomalía:

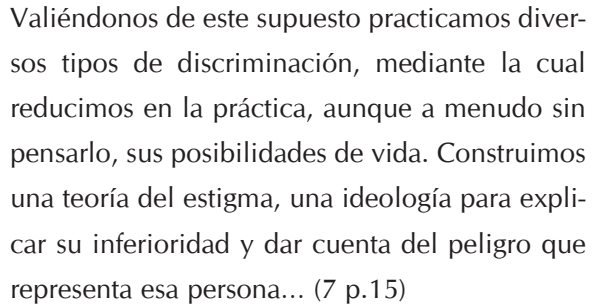

En el contexto de una sociedad medicalizada, el estigma asociado a la transmisión del VIH se convierte en un esquema para distinguir lo considerado normal de lo anormal. Son formas de dominación simbólica: "la dominación simbólica [...] se basa en el desconocimiento y por lo tanto en el reconocimiento de los principios en nombre de los cuales se ejerce" (5 p.170). Su esencia es una función: mantener el control social reproduciendo eso que esconde. Goffman, por ejemplo, señala que el estigma puede escamotear algo más: "racionalizando a veces una animosidad que se basa en otras diferencias, como, por ejemplo, la de clase social" (7 p.15). La consecuencia es que los sujetos "normales" se aparten horrorizados de la senda de las anomalías y refuercen, a través de la discriminación, las barreras de segregación que garantizan el control social. El estigma y la discriminación, entonces, son mecanismos que se complementan y forman parte de lo que Jonathan Mann et al. denominaron en el campo de la transmisión del VIH la otra epidemia (8 p.19), la social, que no se inscribe necesariamente en los procesos biológicos, pero que se ancla en la mirada médica (9): si una persona enferma puede transmitir la enfermedad y los procesos sociales pueden traducirse a metáforas biológicas (sociedades moralmente enfermas, compuestas por personas enfermas de lo mismo, por lo mismo), entonces, las personas enfermas física y socialmente transmiten esa enfermedad en todas las esferas de lo social y son peligrosas. Ante ello, es necesaria una medida higienista: aislar, separar hasta desaparecer al enfermo y la enfermedad. Detrás de estos procesos desacreditadores, excluyentes, invisibilizantes, se ancla el miedo (10) a lo desconocido y a lo incontrolable.

El señalamiento a una persona afectada por una enfermedad incurable, que además se 
identifica con una muerte inmediata que en muchas ocasiones es mejor adelantar con el suicidio (11), constituye una violación al derecho a la dignidad de las personas y a vivir una vida justa, genera sufrimiento y son procesos que conforman inequidades en el campo de la salud pública (12), que desembocan en una mayor desigualdad social (13).

La propuesta del proyecto "Vida Digna" fue terminar con estos atributos negativos y estas formas de exclusión hacia los grupos relacionados con el VIH a partir de intervenciones que incidieran en instancias políticas y de servicios locales. Para ello, se decidió que fuesen las OSC, con o sin registro formal pero con presencia en la comunidad y que estuviesen conformadas por o atendiesen a poblaciones clave, las que impulsaran cambios en los servicios públicos de los niveles municipales y estatales, sobre todo los que concentran a los prestadores de servicios de salud, a la prensa y a la policía. En el año 2005, el proyecto tuvo contacto con 18 OSC; durante sus cuatro años de operación dejaron de participar 9 y se crearon 4 nuevos grupos. Al final del año 2009 participaban 9 OSC. El total de organizaciones que participaron desde el 2005 (año de inicio) al 2009 (año de finalización del proyecto) fue de 18 OSC (Cuadro 1). El proyecto inició su labor realizando "diagnósticos comunitarios participativos", cuyos resultados fueron la base de los planes de trabajo específicos para las necesidades de cada localidad.

Los diagnósticos comunitarios participativos son una metodología de exploración de percepciones y necesidades basada en talleres y sesiones de grupo, en los que se abordan temáticas diversas a través de las experiencias subjetivas de los participantes, para luego integrar sus resultados en una discusión abierta. Para ello, se utilizaron diversas técnicas: la construcción de mapas de la comunidad, redes sociales y del cuerpo; la construcción de un "personaje típico", una bitácora semanal o cotidiana, una línea de vida y un personaje "clave" en la familia; además de una valoración de los servicios a los que pueden acudir y de sus necesidades de salud. En todos estos ejercicios afloró la experiencia personal en experiencias estigmatizadoras y discriminatorias. Se priorizaron los resultados de esta exploración, que dieron lugar a un diagnóstico amplio de necesidades, a partir del cual se iniciaron, en cada localidad, las acciones específicas enmarcadas en el proyecto contra el estigma y la discriminación.

\section{METODOLOGÍA}

Para conocer los procesos específicos que se llevaron a cabo al interior de las organizaciones para implantar el proyecto, se realizaron 26 entrevistas semiestructuradas a representantes de las 9 OSC participantes hasta el término del proyecto, al personal de los servicios de salud locales y a informantes de las poblaciones clave beneficiarias en la ciudad de Santiago de Querétaro (estado de Querétaro); las ciudades de Guanajuato, Irapuato y León (estado de Guanajuato); la ciudad de Aguascalientes (estado de Aguascalientes); y la ciudad de San Luis Potosí, (estado de San Luis Potosí). Los contactos se establecieron con la colaboración de la organización Colectivo Sol. El levantamiento de los datos cualitativos en campo se realizó entre octubre y diciembre de 2009, con una segunda etapa para completar la información en febrero de 2010. Además, se utilizaron como fuentes de observación documental, los planes de trabajo de las organizaciones del año 2008 y sus informes trimestrales del 2009. La información cualitativa sirvió, en este caso, para documentar los procesos por los que transitaron las OSC y para conocer el impacto no mensurable de las acciones del proyecto en las poblaciones atendidas por las OSC.

Para llevar a cabo la comparación de capacidades, tanto de cada organización como de las intervenciones en su conjunto, partimos de la idea de que los cambios observados en las OSC constituyen un indicador importante del impacto que tuvo el proyecto para reducir el estigma y la discriminación ligados a la transmisión del $\mathrm{VIH}$. Ello significa que los resultados de la medición de estas capacidades y su comparación en el tiempo, antes y después de la participación de cada organización, constituyen indicadores de este impacto: si se fortalecieron las capacidades de las organizaciones, se logró un cambio positivo para abatir el estigma y la discriminación en El Bajío, aunque se trate de relaciones indirectas. 
Cuadro 1. Organizaciones participantes en el proyecto "Vida Digna" según trayectoria, poblaciones clave beneficiarias y principales acciones. El Bajío, México. 2005-2009.

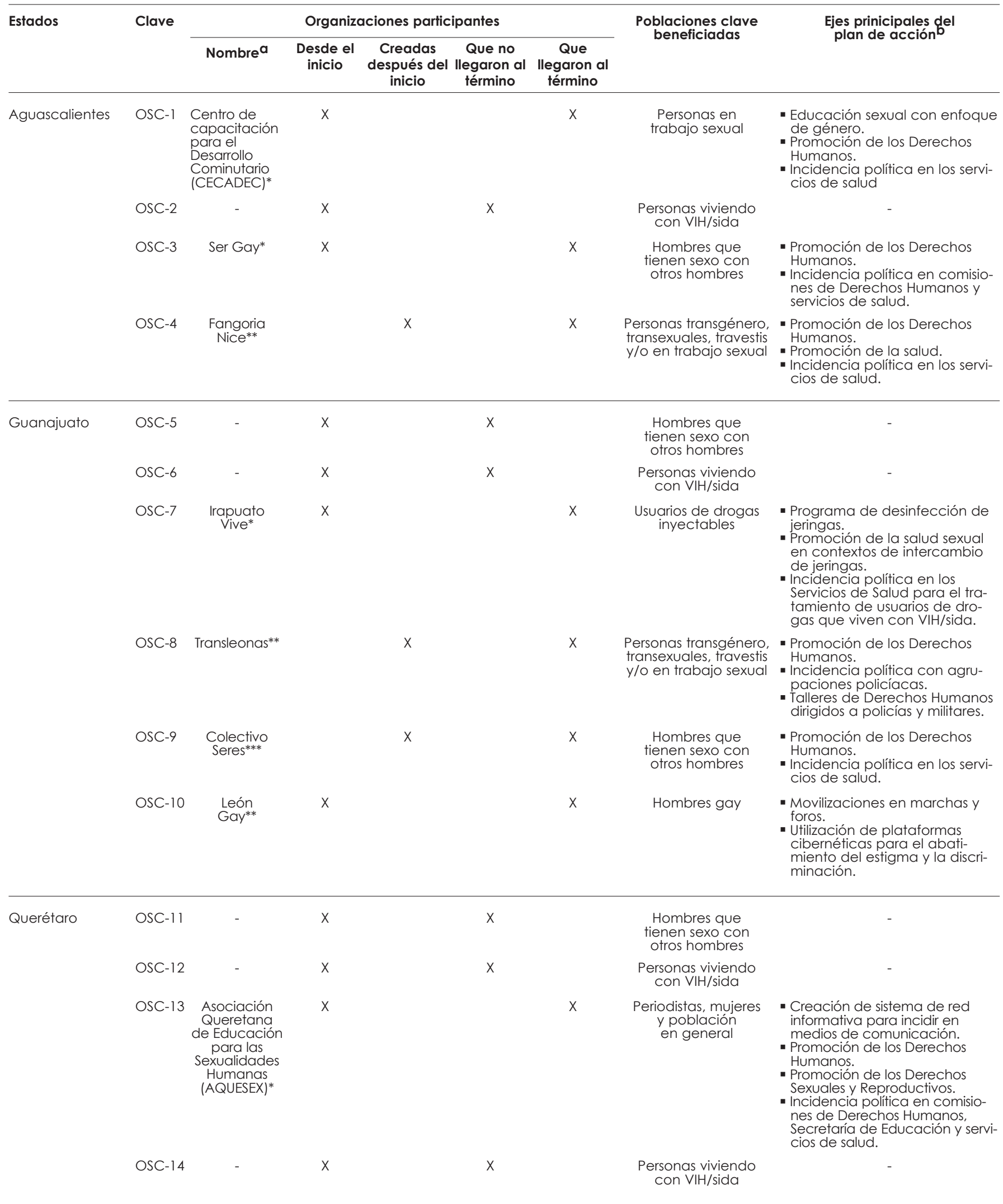


Cuadro 1. Continuación

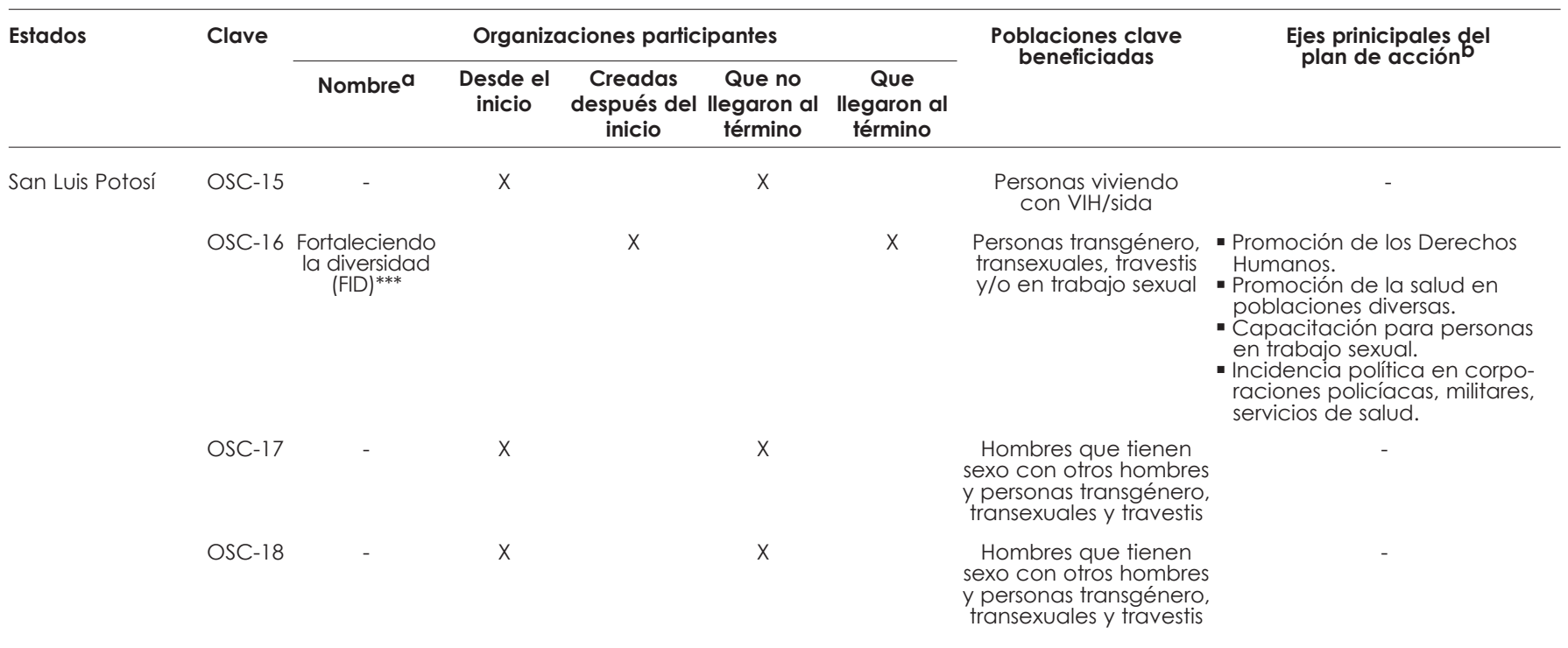

Totales

18

14

9

Fuente: Elaboración propia.

asolo las organizaciones que participaron hasta 2009 accedieron a publicar su nombre.

bsolo de aquellas que participaron hasta 2009.

*Asociaciones civiles constituidas legalmente antes de su participación en el proyecto.

**Organizaciones comunitarias de base, no constituidas legalmente hasta 2009.

***Asociaciones civiles que se constituyeron legalmente durante su participación en el proyecto.

Los cambios en las capacidades se obtuvieron con dos mediciones transversales a través de una encuesta: una basal en el año 2005 y otra en el año 2009, cuando finalizó el proyecto. La información cuantitativa de 2005 fue recopilada por la organización Colectivo Sol al inicio de la intervención. Para recabar la información cuantitativa del año 2009, se diseñó un cuestionario para ser contestado en línea. Este cuestionario contenía las mismas preguntas que la encuesta basal, para permitir la comparación, y se le agregaron otras como complemento. Se invitó a los representantes de las organizaciones participantes para que contestaran la encuesta. A cada organización se la dotó de una clave única para entrar a la plataforma y así salvaguardar la confidencialidad de los participantes; además, el formato en línea contaba con un filtro de inicio, por el cual se explicaban los objetivos de la evaluación y se garantizaba el uso exclusivo de la información para fines científicos y la salvaguarda de la confidencialidad, a menos que la organización estuviese de acuerdo en la publicación de su nombre, lo que constituyó el consentimiento informado. En el año 2009 la encuesta fue contestada por 10 organizaciones pero los cambios en el desempeño integral por capacidad desarrollada por el proyecto solo se pueden analizar en las 8 OSC que respondieron ambas encuestas (2005 y 2009).

Para evaluar los cambios en las organizaciones, se agruparon y analizaron cuatro capacidades que deberían desarrollarse como resultado de la participación en "Vida Digna":

1) La relacionada con los procesos internos de la organización;

2) la capacidad vinculada con el desarrollo de proyectos sobre estigma y discriminación;

3) las capacidades concernientes a lograr la incidencia política en sus localidades; por ejemplo cambios en las legislaciones locales para aumentar el acceso a los servicios de salud, para disminuir las sanciones policíacas por el ejercicio del trabajo sexual en la calle, para crear programas de distribución de condones en las clínicas familiares, etc.; 
4) la capacidad de identificar el abatimiento del estigma y la discriminación como parte importante de las acciones políticas que deben realizar las OSC (Cuadro 2).

Cada indicador se desglosó en variables y se le asignó un puntaje ideal que sirviera de punto de comparación, siguiendo la propuesta weberiana del tipo ideal (Cuadro 2). Para la capacidad 1 (relacionada con los procesos de organización interna) y la 3 (que busca incidir en el ámbito político local), la puntuación fue binaria: 1 si había desarrollado la variable y 0 si contestaba de forma negativa.

Para los indicadores 2 y 4 se operacionalizó la noción de estigma desarrollada por Aggleton y Parker consistente en atributos profundamente desacreditadores que se anclan en estructuras de género, económicas, raciales y sexuales y que se echan a andar y se profundizan en el contexto de la transmisión del VIH (4) (Cuadro 2). El puntaje para estos indicadores se definió como alto, medio o bajo:

- Alto: la definición señala al estigma como compuesto por atributos desacreditadores y toma en consideración los cuatro o tres puntos de anclaje señalados por Aggletton y Parker (4): desigualdades e inequidades por género, económicas, raciales y sexuales. Asimismo, identifica al estigma como barrera para la elaboración de políticas públicas y para el acceso a los servicios.

- Medio: la definición señala al estigma como compuesto por atributos desacreditadores y hace referencia de dos o uno de los anclajes señalados por los autores. Identifica una sola barrera para la elaboración de políticas públicas y para el acceso a los servicios.

- Bajo: solo refiere desacreditación, no identifica obstáculos.

Solo a los fines de aportar claridad en la exposición de los cambios atribuidos al proyecto, las puntuaciones se convirtieron a porcentajes.

Dado que los datos de la encuesta en línea fueron registrados por cada organización, es decir, se trató de un autorreporte, el abordaje cualitativo y la revisión de informes periódicos sirvió también para limitar este sesgo. Este tipo de estudios mixtos, que utilizan la triangulación metodológica, han venido tomando fuerza en los últimos 20 años, entre diversos investigadores, principalmente de las ciencias sociales (16).

En síntesis, el diseño mixto permitió contar con descripciones de los procesos que experimentaron las organizaciones en El Bajío, las cuales complementan y explican los cambios en los índices de sus capacidades.

\section{RESULTADOS}

El proyecto "Vida Digna" tuvo como característica importante atender las necesidades específicas de cada localidad de El Bajío, las cuales fueron detectadas a través de un instrumento denominado "diagnóstico comunitario participativo" que cada grupo aplicó en su entorno. Con base en esos diagnósticos, cada OSC desarrolló su propio plan de acción, que resultó en una variedad de iniciativas. Por ejemplo, en Querétaro, la OSC-13 implantó una intervención directa en los medios de comunicación, consistente en el envío de noticias y comentarios relacionados con la homofobia, la discriminación o la estigmatización de poblaciones clave a los medios impresos a través de Internet. Su diagnóstico partió de una experiencia traumática: el asesinato de uno de sus integrantes y la campaña de homofobia y desprestigio, orquestada desde el gobierno estatal y secundada por los medios de comunicación y la iglesia católica, para justificar las deficiencias de la investigación policíaca. Esa misma OSC organizó talleres sobre conocimientos para evitar el estigma y la discriminación asociados al VIH y a las poblaciones clave dirigidos a periodistas locales, lo que permitió la creación de un nuevo vocabulario en torno a las noticias relacionadas con el VIH y estos grupos. Además, desarrolló materiales electrónicos, sin embargo el mantenimiento de sus páginas les resultó oneroso y de difícil manejo técnico.

Por su parte, en los estados de Aguascalientes, San Luis Potosí y Guanajuato, tres mujeres líderes entre la población transgénero iniciaron actividades; dos de ellas al cobijo de asociaciones formales: la OSC-4 (agrupación comunitaria que nace del trabajo de la 
Cuadro 2. Indicadores, variables y puntuación asignada para la evaluación de capacidades de las organizaciones de la sociedad civil (OSC). El Bajío, 2005-2009.

\begin{tabular}{|c|c|c|}
\hline Indicadores & Variables & Puntuación máxima (ideal): 24 \\
\hline $\begin{array}{l}\text { capacidades para } \\
\text { el desarrollo de } \\
\text { proyectos sobre el } \\
\text { estigma y la } \\
\text { discriminación }\end{array}$ & $\begin{array}{l}\text { Humanos de las poblaciones clave. } \\
\text { 2. Nivel de empoderamiento para enfrentar situaciones de estigmatización, discriminación y } \\
\text { violación de derechos. } \\
\text { 3. Capacidad para gestionar y desarrollar proyectos en la temática de estigma y discriminación. }\end{array}$ & $\begin{array}{l}\text { Alto: } 2 \text { puntos; medio: } 1 \text { punto; } \\
\text { bajo: } 0 \text { puntos }\end{array}$ \\
\hline $\begin{array}{l}\text { III. Capacidad } \\
\text { para la incidencia } \\
\text { política }\end{array}$ & $\begin{array}{l}\text { 1. Desarrollo de actividades con población clave. } \\
\text { 2. Se involucran con consejos estatales para tratamiento y control del VIH/sida, centros ambula- } \\
\text { torios para la prevención y atención del VIH/sida y de las infecciones de transmisión sexual, } \\
\text { comisiones estatales de Derechos Humanos, legisladores u actores políticos clave en la } \\
\text { comunidad. } \\
\text { 3. Identifican la importancia de algunas otras estrategias de incidencia política. } \\
\text { 4. Reconocen y utilizan el uso de herramientas de Diagnóstico Comunitario Participativo (DCP). }\end{array}$ & Puntuación máxima (ideal): 4 \\
\hline \multirow{2}{*}{$\begin{array}{l}\text { IV. Identificación } \\
\text { del estigma y su } \\
\text { relación con la } \\
\text { incidencia } \\
\text { política }\end{array}$} & \multirow{2}{*}{$\begin{array}{l}\text { 1. Definición de estigma. } \\
\text { 2. Identifica al estigma como barrera para la elaboración de políticas públicas y para el acceso } \\
\text { a los servicios. } \\
\text { 3. Identifica la incidencia política y la relaciona con la reducción del estigma. }\end{array}$} & Puntuación máxima (ideal): 6 \\
\hline & & $\begin{array}{l}\text { Alto: } 2 \text { puntos; medio: } 1 \text { punto; } \\
\text { bajo: } 0 \text { puntos }\end{array}$ \\
\hline
\end{tabular}

Fuente: Elaboración propia.

asociación OSC-3) y la OSC-16 (cuya agrupación de origen fue la OSC-15 y la OSC-8), la cual logra su formalización bajo la figura de asociación civil, mientras las otras dos (OSC-4 y OSC-8) continúan como agrupaciones de base, con una fuerte presencia en la comunidad de mujeres transgénero, anteriormente invisibilizadas y perseguidas en sus localidades (Cuadro1).

Trabajar en el mundo de las personas transgénero significa un arduo esfuerzo por parte de las organizaciones. Los nichos de empleo de las chicas trans, como se denominan a sí mismas, son escasos: peluquerías, discotecas y bares o los circuitos de trabajo sexual. Dado que realizan cambios en su cuerpo no pueden pasar desapercibidas y son fácilmente expulsadas de las escuelas, o saben de antemano que ese no es un lugar apropiado para ellas por la violencia con que se las trata allí durante su niñez y su adolescencia. Según sus testimonios, las mujeres transgénero sufren más acoso que la población gay, que al menos tiene el recurso de mantener sus preferencias sexuales en el anonimato, como una forma de defenderse ante el estigma:
A los chicos gays, no se les nota ningún cambio, si ellos así lo desean pueden pasar por chicos "normales" y ello hace que sus posibilidades de estudiar, de formarse, sean mayores que nosotros las trans que desde pequeñas nos vestimos diferente, hablamos diferente, nos movemos diferente, y eso hace que en la escuela nos rechacen, nos persigan, nos salimos de allí. (02 mujer transgénero)

La falta de acceso a las escuelas hace que las capacidades para tareas técnicas se limiten. Por ejemplo, esta investigación solicitaba las respuestas de la encuesta en línea; sin embargo, fue en estas organizaciones donde más se dificultó la recolección de datos por la falta de experiencia en el manejo de Internet y las dificultades en la lecto-escritura.

Los testimonios de representantes de organizaciones y beneficiarios coincidieron en describir las dificultades del trabajo con las chicas trans sobre todo cuando se ubican en los circuitos de sexo comercial. Allí la vulnerabilidad se agudiza debido a la clandestinidad y la persecución de 
estas actividades. Las adicciones, la violencia entre géneros y/o proveniente del narcotráfico, hacen que el trabajo de difusión de información, las acciones de prevención y control para las mujeres transgénero se dificulte. Además, los horarios del trabajo sexual, generalmente nocturno, impiden la participación en talleres y reuniones diurnas; lo mismo ocurre con los horarios para los servicios de salud, que en México nunca están disponibles después de las dos de la tarde, lo que se convierte en una barrera para acceder a información sobre prevención o a condones gratuitos. Aun así, con estas condiciones, estas nuevas agrupaciones han logrado un trabajo sistemático con esta población clave y la OSC-16 obtuvo de parte de la Organización de las Naciones Unidas, en el año 2008, el premio "Lazo Rojo", como reconocimiento a su labor en este difícil campo. Otra organización, la OSC-3, también obtuvo posteriormente ese mismo premio en el año 2010 por su tarea sostenida de defensa de los Derechos Humanos y su lucha contra la estigmatización y discriminación de las poblaciones clave. Actualmente las tres agrupaciones de mujeres transgénero constituyen interlocutores importantes en el desarrollo de políticas relacionadas con el VIH y las poblaciones clave, en su región. En el caso de la organización potosina OSC-16 convocó y reunió en talleres a personas en trabajo sexual, cuerpos policíacos y militares y logró un acuerdo para detener la persecución de las personas en los circuitos de servicio sexual.

En Aguascalientes, por su parte, el trabajo conjunto de la OSC-1, la OSC-3 y la OSC-4, logró una fuerte incidencia política en los organismos estatales de Derechos Humanos y en el Congreso local, lo que ha vuelto visibles a las poblaciones tanto de hombres gay como de personas transgénero y/o en trabajo sexual, diferenciándolos, por un lado, pero reconociendo su vulnerabilidad ante la discriminación, la estigmatización, las violaciones a sus derechos y las barreras al acceso a los servicios de salud.

En el estado de Guanajuato, la OSC-8 fundada a partir del proyecto, ha hecho un trabajo de promoción del condón y de incidencia política para cambiar leyes locales que estigmatizan a las personas en trabajo sexual, lo que incluye a mujeres transgénero, logrando el respeto de los espacios de trabajo, amortiguando así la persecución de policías o de agentes sanitarios. La OSC-8 en conjunto con la OSC-10 han organizado marchas y eventos que visibilizan la problemática del VIH, la discriminación y la estigmatización a sus poblaciones clave. En el mismo estado de Guanajuato, en la ciudad de Irapuato, la OSC-7 logró realizar una efectiva campaña de desinfección de jeringas y mitigación del daño causado por el VIH entre usuarios de drogas inyectables, cuya criminalización impedía su acceso a los servicios de salud públicos y los mantenía en condiciones de clandestinidad. A partir de un trabajo entre pares, la organización ha logrado abrir el acceso a los servicios estatales públicos de salud para brindar diagnósticos y tratamientos antirretrovirales a los usuarios de drogas inyectables y así detener la transmisión del VIH en ese grupo. Es la primera iniciativa que realiza un trabajo entre estos grupos -más allá de las ciudades fronterizas del norte de la República Mexicana- y que reconoce su existencia en la región central de México. Además, como parte de sus trabajos de apoyo a otros grupos clave, el proyecto "Vida Digna" estimuló la creación de la OSC-9, una nueva organización en la ciudad de Guanajuato, para brindar atención a los hombres gay, dado que allí no se contaba con ninguna representación de este grupo. La visibilización de los grupos de hombres gay en esta ciudad posibilitó que las campañas de distribución de condones se fortalecieran y que los prestadores de servicios de salud se sensibilizaran para evitar la discriminación ante la solicitud de pruebas rápidas y/o tratamientos antirretrovirales.

A partir de los datos obtenidos en las dos encuestas realizadas en 2005 y 2009 se compararon las capacidades de 8 organizaciones participantes (Cuadro 3). Esta comparación sirvió para medir las capacidades del proyecto en forma agregada, es decir, las funciones que desarrolló "Vida Digna". Esos cálculos se llevaron a cabo con los resultados de las 8 organizaciones que contaban con medición basal (2005) y medición final (2009).

El abordaje cualitativo permitió observar que las organizaciones identifican claramente a los actores políticos con quienes pueden establecer alianzas para la formulación de políticas públicas. Además, se logró una concientización de la importancia de las acciones a largo plazo a partir de la búsqueda de financiamientos externos para otros proyectos: 
Cuadro 3. Comparación de capacidades de las organizaciones participantes. El Bajío. 2005-2009.

\begin{tabular}{|c|c|c|c|c|c|c|c|c|c|c|c|c|c|c|}
\hline \multirow[b]{2}{*}{ Clave } & \multirow[b]{2}{*}{ Nombre } & \multirow[b]{2}{*}{ Estado } & \multicolumn{6}{|c|}{ Capacidades 2005} & \multicolumn{6}{|c|}{ Capacidades 2009} \\
\hline & & & 1 & II & III & IV & $\begin{array}{l}\text { Puntaje } \\
\text { Ideal: } 24\end{array}$ & $\begin{array}{l}\text { Capacidades } \\
\text { alcanzadas } \\
\%\end{array}$ & 1 & II & III & IV & $\begin{array}{l}\text { Puntaje } \\
\text { Ideal: } 24\end{array}$ & $\begin{array}{c}\text { Capacidades } \\
\text { alcanzadas } \\
\%\end{array}$ \\
\hline OSC-9 & Colectivo Seres & Guanajuato & 8 & 5 & 2 & 3 & 18 & 75,0 & 8 & 6 & 4 & 5 & 23 & 95,9 \\
\hline OSC-13 & AQUESEX & Querétaro & 7 & 5 & 3 & 4 & 19 & 79,2 & 7 & 6 & 4 & 5 & 22 & 91,7 \\
\hline OsC-3 & Ser gay & Aguascalientes & 3 & 4 & 4 & 5 & 16 & 66,7 & 7 & 6 & 4 & 5 & 22 & 91,7 \\
\hline OSC-7 & Irapuato Vive & Guanajuato & 7 & 5 & 3 & 3 & 18 & 75,0 & 7 & 6 & 4 & 3 & 20 & 83,3 \\
\hline OSC-1 & CECADEC & Aguascalientes & 6 & 5 & 3 & 4 & 18 & 75,0 & 6 & 6 & 3 & 5 & 20 & 83,3 \\
\hline OSC-14 & COIVHIS & Querétaro & 0 & 0 & 0 & 4 & 4 & 16,7 & 4 & 5 & 4 & 4 & 17 & 70,8 \\
\hline OSC-4 & Fangoria Nice & Aguascalientes & 2 & 3 & 1 & 0 & 6 & 25,0 & 2 & 3 & 3 & 4 & 12 & 50,0 \\
\hline OSC-8 & Transleonas & Guanajuato & 0 & 3 & 2 & 0 & 5 & 20,8 & 2 & 3 & 3 & 3 & 11 & 45,8 \\
\hline Totales & & & 33 & 30 & 18 & 23 & 104 & 54,2 & 43 & 41 & 29 & 34 & 147 & 76,6 \\
\hline
\end{tabular}

Fuente: Elaboración propia a partir de Encuesta basal proyecto"Vida Digna", 2005, y Encuesta en línea "Evaluación del proyecto Vida Digna", Noviembre de 2009-febrero de 2010. Cuernavaca, Morelos, México.

Nota: No se incluyen los datos de las OSC-10 y OSC-16 para no alterar la comparación con el año 2005.

| = Estructura y procesos internos. II = Conocimientos y capacidades para el desarrollo de proyectos sobre estigma y discriminación. III = Capacidad de incidencia política. IV = Identificación del estigma y su relación con la incidencia política.

AQUESEX = Asociación Queretana de Educación para las Sexualidades Humanas. CECADEC = Centro de Orientación e Información de $\mathrm{VIH} /$ sida. COIVHIS = Centro de Capacitación para el Desarrollo Comunitario.

Yo pienso que es un proyecto que debe continuarse, porque [...] ha dado cohesión a todas las organizaciones civiles. Los recursos te permiten generar un trabajo unificado. Si se pierde el financiamiento las organizaciones se van a separar $y$ van a perder su peso, que de alguna forma se han ido ganando. (Informante, OSC-9, Guanajuato)

Asimismo, el proyecto logró un impacto importante en la visibilización de poblaciones estigmatizadas y mejoró el acceso a los servicios de salud de grupos alejados de estos por la estigmatización:

\footnotetext{
Actualmente un médico ausculta a gays en su consultorio, cuando antes ni los tocaba; el temor al contagio casual era más generalizado, sobre todo en enfermeras, mientras que ahora se encuentra más focalizado y es más fácil de localizar y atacar. (Informante, OSC-3, Aguascalientes)
}

La violencia que genera el rechazo a las poblaciones clave pudo detenerse a partir de este proyecto. Según los testimonios, se logró un impacto positivo en la población en general:
Ahora desde que está [la OSC-16] ha cambiado mucho [el ambiente de trabajo], favorablemente. Si no estuviera [la organización], iban y hacían y deshacían con nosotras. [...] Antes había mucho irrespeto, nos decían de cosas, nos querían hasta humillar, nos aventaban cosas: basura, botes de cerveza, botellas, nos querían hasta manosear. Entonces ahora a partir de que ha llegado [la OSC-16], ha cambiado todo, porque ahora si pasa una patrulla y hay un borrachito que nos quiere molestar, nos apoya, y antes no, antes se iban derecho. Ya cesaron todo ese tipo de discriminación, porque era mucha discriminación la que sufríamos, nos discriminaban bastante, porque ahora ya nos respetan. Hemos tenido pláticas, por parte de Censida, por parte de [la organización]. (Persona en circuito de trabajo sexual, San Luis Potosí)

El proyecto mitigó, aunque quizá solo temporalmente, uno de los problemas de violencia más generalizada que padecen las poblaciones clave en México, sobre todo las relacionadas con circuitos de trabajo sexual: el acoso policíaco y militar. 
Hace dos años a mí me pasó que me golpearon los policías a dos muchachas. A la entrada, no las dejaron entrar [a la Zona de Tolerancia]: "no entres", "es que le voy a pedir para el taxi" [a la dueña del bar]. Entonces ellas querían que yo les diera para el taxi, y no las dejaron entrar, las golpearon, las golpearon muy, muy feo. Entonces yo tenía mucho coraje. Entonces yo pensé que no me tenía que dejar. Te digo, afortunadamente llegaron en buen momento [la OSC-1]. Me empezaron a orientar y yo me empecé a defender más. Ellos [los policías] vieron, porque cuando vieron que llegaron ellas [la OSC-1], empezaron a dejar de violar nuestros derechos, a dejar de discriminarlas. Por ejemplo a las muchachas, les decían: "si no trajiste tus análisis vete, no trabajas". Ahora no, si no traen sus fotos por ejemplo, no hay problema, para la otra semana. Pero comprometiéndose y hablando con nosotros. Ya hay comunicación. Ya las dejan salir [de la Zona de Tolerancia]. Entonces nos orientaron [la OSC1] para que defendiéramos a nuestras muchachas y nuestros derechos. (Dueña de bar, Aguascalientes)

Con el abordaje cuantitativo se logró documentar y medir los incrementos en las capacidades de las 8 organizaciones que respondieron a las encuestas realizadas en 2005 y 2009 (Cuadro 4). La OSC-9, fundada a partir del proyecto, fue la organización con mayor fortalecimiento (de 18 puntos en 2005 pasó a 23 puntos en 2009), y aunque la OSC-4 y la OSC-8 están en los últimos lugares (25 a 50 puntos y 20,8 a 45,8 respectivamente) sus cambios resultan importantes pues se trata de organizaciones de mujeres transgénero, las cuales no se encontraban representadas en los grupos organizados de la sociedad civil relacionados con el control y prevención del VIH. Al agrupar las capacidades (Cuadro 4), se observa también un fortalecimiento general del proyecto si comparamos los resultados del año 2005 con el año 2009. La capacidad más desarrollada fue la de incidencia política (18 puntos contra 29), le siguieron el desarroIlo de conocimientos para proyectos sobre estigma y discriminación (30 a 41); la identificación de la disminución del estigma como parte importante de las políticas públicas (de 23 a 34 puntos) y, por último, el fortalecimiento de estructuras y procesos internos (33 a 43 puntos). En total, estas capacidades pasaron de 104 a 147 puntos (Cuadro 4).

\section{CONCLUSIONES}

Si bien el proyecto aumentó las cuatro capacidades para la reducción del estigma y la discriminación en las organizaciones de El Bajío, al pasar de un $54,2 \%$ en el año 2005 a un $76,6 \%$ en el año 2009, al volver visibles a grupos históricamente estigmatizados y al posicionarse en las instancias estatales y nacionales a partir de labores de incidencia política, tuvo un menor desempeño en el desarrollo de acciones para fortalecer las estructuras internas de las organizaciones. El no crecer tanto en este rubro tiene consecuencias en la permanencia a largo plazo de las acciones contra el estigma y la discriminación pues la consolidación de la estructura interna de las organizaciones permite también su reconocimiento externo, sobre todo de entes gubernamentales o agencias, para financiar sus proyectos de intervención, bolsas de financiamiento importantes para seguir operando a pesar de la finalización del proyecto.

Cuadro 4. Desempeño integral de la intervención para abatir el estigma y la discriminación. El Bajío, 2005 y 2009.

\begin{tabular}{|c|c|c|c|c|}
\hline \multirow[t]{2}{*}{ Capacidades } & \multicolumn{2}{|c|}{ Puntuaciones } & \multicolumn{2}{|c|}{ Porcentajes } \\
\hline & 2005 & 2009 & 2005 & $\begin{array}{c}2009 \\
\%\end{array}$ \\
\hline $\begin{array}{l}\text { I. Estructura y procesos internos. } \\
\text { Puntuación máxima: } 64\end{array}$ & 33 & 43 & 51,6 & 67,2 \\
\hline $\begin{array}{l}\text { II. Conocimientos y capacidades } \\
\text { para el desarrollo de proyectos } \\
\text { sobre estigma y discriminación. } \\
\text { Puntuación máxima: } 48\end{array}$ & 30 & 41 & 62,5 & 85,4 \\
\hline $\begin{array}{l}\text { III. Capacidad de incidencia política. } \\
\text { Puntuación máxima: } 32\end{array}$ & 18 & 29 & 56,2 & 90,6 \\
\hline $\begin{array}{l}\text { IV. Identificación del estigma y su } \\
\text { relación con la incidencia política. } \\
\text { Puntuación máxima: } 48\end{array}$ & 23 & 34 & 47,9 & 70,8 \\
\hline $\begin{array}{l}\text { Totales } \\
\text { Puntuación máxima= } 192\end{array}$ & 104 & 147 & 54,2 & 76,6 \\
\hline
\end{tabular}

Fuente: Elaboración propia a partir de Encuesta basal proyecto"Vida Digna", 2005, y Encuesta en línea "Evaluación del proyecto Vida Digna", Noviembre de 2009-febrero de 2010. Cuernavaca, Morelos, México. 


\section{Organizaciones de personas transgénero en circuitos de trabajo sexual}

Especial atención merece el logro del proyecto al consolidar el trabajo de las tres organizaciones que enfocaron sus acciones a las personas transgénero, quizá el grupo clave más señalado negativamente y discriminado debido a las prácticas en torno a sus cambios corporales y por su entrada y salida de circuitos de trabajo sexual. Todas las agrupaciones enfocadas a estos grupos se crearon bajo el impulso del proyecto y están dirigidas por mujeres transgénero en trabajo sexual. La OSC-4 y la OSC-8 duplicaron sus capacidades a pesar de las fuertes limitaciones y de los procesos de estigmatización y discriminación a las que han estado sujetas históricamente. A pesar de estos cambios positivos, podemos señalar que estas dos organizaciones, al no lograr su constitución legal corren el riesgo de no especializarse en las labores de incidencia política y dispersarse en una defensa inmediata sin cambios estructurales del estigma y la discriminación. Además, el análisis cualitativo nos indica que la OSC-16, que se constituyó legalmente, coadyuvó a la creación de nuevos nichos de trabajo para las personas en circuitos de trabajo sexual, que históricamente han visto reducidas sus oportunidades laborales debido a la estigmatización que ahonda la pobreza y la explotación, por lo que nos atreveríamos a afirmar que la consolidación legal de las organizaciones también significa oportunidades de empleo para grupos marginados de la esfera laboral.

\section{Conocimientos sobre estigma y discriminación: obstáculos para su práctica}

En general, todas las organizaciones tienen en claro el concepto de estigma y discriminación. Además, los representantes de las organizaciones participantes hasta el 2009 señalan claramente que el proyecto les ayudó a reconstruir su identidad desde un lugar diferente a la estigmatización, ya que muchos de estos líderes son al mismo tiempo beneficiarios de sus propias organizaciones. Sin embargo, el abordaje cualitativo permitió documentar que todas las organizaciones refieren no haber superado completamente la homofobia al interior y entre los grupos diversos. Si bien los participantes en el proyecto identifican los principales estigmas preexistentes, no es clara la reflexión sobre la diferenciación de los mismos al momento de ponerlos a operar para llevar a cabo intervenciones concretas para reducirlos, lo que ha traido como consecuencia la atomización de las acciones conjuntas entre las organizaciones de la misma región.

\section{Indicadores de la evaluación y la movilidad de las organizaciones}

El proyecto constituyó una intervención eficaz en el fortalecimiento de las OSC participantes: consolidó algunas y fundó nuevas. Sin embargo, el registro y comparación de resultados se dificultó, dada la diversidad de acciones llevadas a cabo. Esto señala, otra vez, la importancia de desarrollar índices y técnicas de registros de acciones y su impacto desde el inicio de las intervenciones que además den cuenta de sus especificidades. El uso de herramientas de Internet funcionó para esta evaluación, por lo que se hace necesario revisar y retomar esta experiencia para otros trabajos similares.

Por otro lado, Ilama la atención la movilidad de las organizaciones participantes en el proyecto durante sus cinco años de operación. De las 14 OSC iniciales, 5 se mantuvieron durante toda su operación, una participó de forma intermitente y se fundaron 4. La evaluación permitió registrar que la mayoría de los casos de dispersión de las OSC estuvieron asociados a la tendencia a la clandestinidad propia de las poblaciones clave, que se contraponía a los objetivos de visibilización que planteaba el proyecto. La discusión de esa contradicción se hace impostergable en cualquier intervención con grupos estigmatizados y/o criminalizados.

La necesidad de evaluar cambios en los conocimientos, las percepciones y las prácticas en el personal que brinda servicios de salud a estas poblaciones hace también necesario revisar la pertinencia de aplicar pequeños cuestionarios ya validados sobre estigma y discriminación a dichos prestadores.

En resumen, podemos señalar, con base en las evidencias arrojadas por esta evaluación, 
que las intervenciones para abatir el estigma y la discriminación:

1) Son exitosas a través de organizaciones desde y para la sociedad civil. El trabajo entre pares, como fueron las acciones Ilevadas a cabo con usuarios de drogas inyectables, contribuye a una mayor confianza para acceder a poblaciones en la clandestinidad o invisibles en el plano de lo social y puede incidir políticamente en la construcción de programas que respondan a necesidades específicas.

2) Deben sustentarse en el diseño de planes estratégicos que dirijan el trabajo de las organizaciones. El fortalecimiento de los procesos internos de las organizaciones y su constitución formal constituyen un elemento importante para garantizar su sustentabilidad.

3) Implican la construcción de indicadores de proceso que sean comparables entre sí pero que a la vez den cuenta de las especificidades de programas como "Vida Digna" que atendieron situaciones particulares. El estigma y la discriminación adquieren diferentes formas debido a las variaciones del contexto por lo que la construcción de indicadores para evaluar su impacto significa el reto de uniformar sin desdibujar.

4) Necesitan del desarrollo de capacidades técnicas para el manejo de información en la Web. Se debe aprovechar la capacidad existente a través de las Ilamadas "redes sociales" con el fin de disminuir costos para la comunicación y difusión de acciones y permitir a las organizaciones focalizar sus recursos en el abatimiento del estigma y la discriminación y no en la administración de páginas electrónicas costosas.
5) Deben desarrollar capacidades para que las organizaciones conozcan de las dinámicas de los líderes locales, de la organización interna de los servicios de salud y de los medios de comunicación, con el fin de hacer más precisas y eficientes sus intervenciones en el campo de la política. Los mapas de instituciones, la construcción de redes de liderazgo y de funciones son necesarias dependiendo del tipo y nivel del plan de incidencia política. No es lo mismo una intervención en las políticas internas de los hospitales para la atención de las poblaciones clave que proponer cambios a nivel jurídico para diseñar normas que disminuyan el estigma y la discriminación.

Por último, nos atrevemos a sugerir que las intervenciones, sobre todo para formar nuevas organizaciones que atiendan a poblaciones altamente estigmatizadas, tendrían que intercambiar experiencias sobre las implicaciones de un cambio en su rol laboral de población clave a representantes de OSC. La fundación de OSC para estas poblaciones permite abrir nuevos nichos de trabajo remunerado en grupos cuyas oportunidades se encuentran limitadas, como lo demostró el caso de las organizaciones enfocadas a trabajar con personas en circuitos de trabajo sexual. Sin embargo, este punto debe ir aparejado con la demanda de reconocer al trabajo sexual como un empleo formal que implica derechos y obligaciones para las personas cercanas a esta actividad. El derecho a la ciudadanía debería guiar esta reflexión, lo cual no ocurre en México.

\section{AGRADECIMIENTOS}

Este texto no hubiera sido posible sin la confianza y el apoyo de la International HIV/AIDS Alliance y del programa Positive Action, organismos internacionales que financiaron el proyecto "Vida Digna". La organización Colectivo Sol fue uno de los ejes más importantes en esta evaluación, ya que a través de Juan Jacobo Hernández, César Coria y su equipo de trabajo nos facilitaron toda la información de forma oportuna, transparente y bondadosa. Asimismo reconocemos los esfuerzos de nuestro experto en diseño de bases de datos en red César Meza Carrillo y la asistencia de Carolina Becerril y Ruy Caseb Aron. Especial mención merecen, por supuesto, las organizaciones participantes en "Vida Digna". A ellas nuestro más sincero reconocimiento por su tenacidad y compromiso en el abatimiento del estigma y la discriminación. 


\section{REFERENCIAS BIBLIOGRÁFICAS}

1. Centro Nacional para la Prevención y Control del VIH/Sida. Gráficas del Censo Nacional de las Organizaciones de la Sociedad Civil [Internet]. México: CENSIDA; 2011 [citado 4 oct 2011]. Disponible en: http://www.censida.salud.gob.mx/ descargas/osc_conv/graficas_censo.pdf.

2. Amigos contra el Sida AC. Listado general [Internet]. México [citado 4 oct 2011]. Disponible en: http://www.aids-sida.org/archivos/directorio_nacional/listadogeneral.html.

3. Prance R. Final Report to GlaxoSmithKline Positive Action Programme: Vida Digna phase 2: reducing HIV/AIDS-related stigma and discrimination in Mexico, January to December. Alliance International HIV/AIDS: 2008.

4. Aggleton P, Parker R. Estigma y discriminación relacionados con el VIH/SIDA: un marco conceptual e implicaciones para la acción. México DF: El Colegio de México; 2002. (Documento de trabajo No 9).

5. Bourdieu P. Razones prácticas. Sobre la teoría de la acción. Barcelona: Anagrama; 1994.

6. Infante C, Zarco A, Cuadra SM, Morrison K, Caballero $M$, Bronfman $M$, Magis C. El estigma asociado al VIH/SIDA: el caso de los prestadores de servicios de salud en México. Salud Pública de México. 2006;48(2):141-150.

7. Goffman E. Estigma: La identidad deteriorada. Buenos Aires: Amorrortu; 2001.

8. Mann J, Gostin L, Gruskin S, Brennan T, Lazzarini Z, Fineberg HV. Health and Human Rights. Health and Human Rights. 1994;1(1):6-23.
9. Foucault M. El nacimiento de la clínica: Una arqueología de la mirada médica. México DF: Siglo XXI Editores; 2001.

10. Cuadra SM, Infante C, Zarco A, Morrison K, Bronfman M. Reporte cualitativo sobre el estigma y la discriminación relacionados con las personas que viven con $\mathrm{VIH}$ : el caso de los proveedores de servicios de salud. Cuernavaca: CISS/INSP; 2003.

11. Cuadra SM. Vulnerabilidades para la transmisión del Virus de Inmunodeficiencia Humana en contextos de movilización: mujeres en trabajo sexual de Chetumal, Quintana Roo. [Tesis de Doctorado]. México DF: Facultad de Ciencias Políticas y Sociales, Universidad Autónoma de México; 2012.

12. Whitehead M. The concepts and principles of equity and health. Copenhagen: World Health Organization; 2000.

13. Silva LV, Almeida NF. Eqüidade em saúde: uma análise crítica de conceitos. Cadernos de Saúde Pública. 2009;25(Supl 2):S217-S226.

14. Rawls J. La justicia como imparcialidad. En: Teoría de la justicia. México DF: Fondo de Cultura Económica; 1995. p. 17-61.

15. Cortina A, Martínez E. El ámbito de la filosofía práctica. En: Ética. Madrid: Editorial Akal; 1996. p. 9-28.

16. Greene JC. Is mixed methods social inquiry a distinctive methodology? Journal of Mixed Methods Research. 2008;2(1):7-22.

\section{FORMA DE CITAR}

Cuadra-Hernández SM, Zarco-Mera A, Infante-Xibillé C, Caballero-García M. La organización de las poblaciones clave ligadas a la transmisión del VIH: una intervención para abatir el estigma; México, 2005-2009. Salud Colectiva. 2012;8(2):191-204.

Recibido el 24 de junio de 2011

Versión final presentada el 4 de octubre de 2011

Aprobado el 28 de octubre de 2011 